\title{
Can MS fully exploit the benefits of fast chromatography?
}

\begin{abstract}
"Using data-dependant or -independent acquisition workflows, modern mass spectrometric instrumentation can easily generate large volumes of data. The challenge is now in the data processing to extract the relevant information to address relevant biological questions."
\end{abstract}

Over the last two decades, LC coupled to MS detection (LC-MS) has become a reference analytical method for qualitative and quantitative analysis of proteomics, food science, pharmaceuticals, toxicology and environmental samples. With regards to LC, most of the applications use reverse phase, normal phase or hydrophilic interaction LC with various configurations from nano-LC $(100 \mu \mathrm{m}$ internal diameter [i.d.] columns) to conventional bore LC (4.6 $\mathrm{mm}$ i.d. columns) including columnswitching setups [1]. While during the early days of LC-MS mainly triple quadrupole mass spectrometers were used, nowadays, a broad range of mass analyzers or hybrids are commonly available. LC-MS has become far more powerful since the recent innovations in MS and improved resolving power in chromatography.

\section{High-resolution MS}

The resolution power of a mass spectrometer can be defined as its ability to distinguish two peaks of slightly different mass-to-charge ratio. While regular quadrupole mass analyzers have unit mass resolution, ion-trap instruments can, by lowering the scan speed, achieve higher resolution power up to 20,000, but with limited mass accuracy. In practice there are two categories of mass spectrometers that can achieve high resolution with good mass accuracies (<5 ppm): TOF-MS and fourier transform (FT)-MS, including the FT ion cyclotron resonance and the Orbitrap. The significant difference between the two types of instruments is the duty cycle. On a FT-MS instrument such as the Orbitrap, working at a resolving power of 10,000 requires a duty cycle of $100 \mathrm{~ms}$ and a resolution power of 100,000 is achieved at the expense of a duty cycle of 1-1.5 s [2]. In the case of a TOF geometry instruments, such as the TripleTOF 5600, the resolving power is not dependent on the duty cycle and the system can acquire data in as little as $10 \mathrm{~ms}$ with a resolving power of 20,000-30,000 [3].

\section{Fast chromatography}

In bionalysis, a significant amount of effort was spent to develop LC-MS assays with runtimes of less than 1 min using short columns $(\sim 10-20 \mathrm{~mm})$ and high liquid flow rates $(500-1000 \mu \mathrm{l} / \mathrm{min})$ [1]. With conventional LC, most of the chromatographic peaks have a peak width at half-height (PWHH) of 10-20 s, while with fast chromatography PWHH is $0.5-3 \mathrm{~s}$. However, this is the cost of separation efficiency. In quantitative work at least 12-15 data points for LC peak integration are needed to achieve good assay precisions and accuracies. Triple quadrupole instruments operated in the selected reaction monitoring (SRM) mode can have a duty cycle in the range of 10 to $50 \mathrm{~ms}$ for each analyte, which is often sufficient for LC peak integration. Progress in column technology, including small particles, monolithic, core shell and temperature-stable materials [4-6], has significantly increased the separation efficiency in LC with very narrow LC peaks $(\mathrm{PWHH}<1$ s). Compared with classical LC, the analysis time can be reduced significantly (typically ten-times) while maintaining the separation efficacy of conventional chromatography. With the acquisition speed in the SRM mode of modern instruments, which can be as low as $1 \mathrm{msec}$, or by using scheduled SRM approaches, the targeted analysis of several hundred compounds can be performed while maintaining the chromatographic performance. The situation is completely different when considering qualitative analysis where, for quadrupole instruments, duty cycle is dependent on the mass range (typically a duty cycle of $500 \mathrm{~ms}$ for a $\mathrm{m} / \mathrm{z}$ range of 1000). In this case,

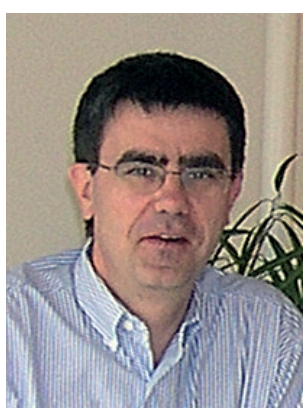

Gérard Hopfgartner Life Sciences Mass Spectrometry, School of Pharmaceutical Sciences, University of Geneva,

University of Lausanne, 30 Quai Ernest Ansermet, $\mathrm{CH}-12$ II Geneva, Switzerland E-mail: Gerard.Hopfgartner@ unige.ch 
a good quality single scan spectra can still be obtained but the chromatographic resolution is destroyed. Ion traps can scan much faster with better sensitivities but the injection time has to also be considered (at lower concentrations, higher injection times are required). In practice, it is challenging to obtain duty cycles below $100 \mathrm{~ms}$ with good sensitivities. Similar duty cycles can be obtained on the Orbitrap, but without fully taking advantage of the resolving power of the instrument. In the case of TOF-based instruments, high acquisition rates are mainly independent of the resolving power and make it an ideal detector for fast chromatography.

\section{"In the case of TOF-based instruments, high acquisition rates are mainly independent of the resolving power and make it an ideal detector for fast chromatography."}

\section{MS versus MS/MS modes}

MS with atmospheric pressure ionization determines the MW of a compound, and by combining the high resolving power with an isotopic distribution fit algorithm, unique elemental formulas can be proposed. However, in many fields, such as drug metabolism, metabolomics and proteomics, this is insufficient and MS/MS plays a key role for structural identification and analyte quantitation. In addition, NMR is unfortunately far less sensitive than MS and, therefore, owing to limited sample amounts, one has to rely solely on MS. Moreover, MS has intrinsically higher throughput capabilities than NMR. Most MS applications use datadependent or information-dependent acquisition workflows using various types of survey scans and generally several product-ion-dependent scans. The MS duty cycle can be of several seconds and only a limited set of precursor ions can be selected to generate product ions spectra. Another approach to maximize the instrument duty cycle is to perform data-independent MS acquisitions by alternating low- and highenergy scans of all the precursor ions entering into the collision cell. Using sophisticated data processing software, product ions, neutral losses and precursor ions, information can be obtained in a single analysis [7]. This procedure is often referred as $\mathrm{MS}^{\mathrm{E}}$ or FragAll and requires highresolving power MS. To perform various MS experiments in a single LC-MS run is particularly attractive for simultaneous qualitative and quantitative analysis [8]. Bioanalytical work is mainly shifting from single-compound quantitative analysis to multiple compounds, thus, qualitative and quantitative analysis with improved chromatographic resolution and fast acquiring mass spectrometers, operating with MS and MS/MS capabilities and mixing dependent and independent acquisition modes of operation are becoming essential. Both workflows are compatible with fast LC, with one (MS $/$ FragAll) offering a more universal setup and the other providing more selective data acquisition, but with the risk of missing some information.

\section{"Bioanalytical work is mainly shifting from single-compound quantitative analysis to multiple compounds..."}

\section{Future perspective}

Using data-dependant or -independent acquisition workflows, modern mass spectrometric instrumentation can easily generate large volumes of data. The challenge is now in the data processing to extract the relevant information to address relevant biological questions. The key parameter that needs to be maximized is the ratio between data quality and data volume. To cope with sample complexity, further hyphenation techniques would be very helpful. Over the last few years, ion mobility MS has become a practicable tool [9]. In differential ion mobility spectrometry (DMS), also known as high-field asymmetric waveform ion mobility spectrometry, ions are separated based on their mobility under high and low electric fields, and therefore it is possible to separate isobaric compounds. Although DMS suffered from poor separation power, the ability to introduce polar modifiers, such as isopropanol, to the gas phase transporting the ions amplifies the high- and low-field mobility difference and therefore significantly improves the resolution [10]. In fact, DMS could also be seen as a gasphase separation technique prior to MS detection and could be used in multidimensional separation setup. In DMS, a compensation voltage can be set at a discrete value or ramped, which could be associated with an isocratic or gradient LC separation mode. The applicability of real-time 2D separation, which can be performed with the first dimension relying on LC and the second dimension relying on DMS, has recently been illustrated. The data obtained during the analysis of complex matrix (e.g., urine) showed that 
the second DMS dimension to improves the data quality without affecting the analysis time and resolves composite MS/MS spectra due to different compensation voltage values for isobaric compounds [11]. However, although real 2D separation (LC-DMS) offers more resolution power, it challenges the acquisition speed and the sensitivity of the mass spectrometer as well as data processing.

\section{Bibliography}

1 Hopfgartner G, Bourgogne E. Quantitative high-throughput analysis of drugs in biological matrices by mass spectrometry. Mass Spectrom. Rev. 22, 195-214 2003.

2 Makarov A, Scigelova M. Coupling liquid chromatography to Orbitrap mass spectrometry. J. Chromatogr. A 1217, 3938-3945 2010.

3 Hopfgartner G, LeBlanc YJC, Varesio E. High resolution liquid chromatography and high resolution mass spectrometry for simultaneous qualitative and quantitative analysis. Presented at: The 58th ASMS Conference on Mass Spectrometry and Allied Topics. Salt Lake City, UT, USA, 23-27 May 2010.

4 Swartz ME. UPLCTM: an introduction and review. J. Liq. Chromatogr. Relat. Technol. 28, 1253-1263 (2005).

\section{Financial \& competing interests disclosure}

The author has no relevant affliations or financial involvement with any organization or entity with a financial interest in or financial conflict with the subject matter or materials discussed in the manuscript. This includes employment, consultancies, honoraria, stock ownership or options, expert testimony, grants or patents received or pending, or royalties.

No writing assistance was utilized in the production of this manuscript.
5 Unger KK, Skudas R, Schulte MM. Particle packed columns and monolithic columns in high-performance liquid chromatography comparison and critical appraisal. J. Chromatogr. A 1184, 393-415 (2008).

6 Wu NJ, Thompson R. Fast and efficient separations using reversed phase liquid chromatography. J. Liq. Chromatogr. Relat. Technol. 29, 949-988 (2006).

7 Plumb RS, Johnson KA, Rainville P et al. UPLC/MSE: a new approach for generating molecular fragment information for biomarker structure elucidation. Rapid Commun. Mass Spectrom. 20, 1989-1994 (2006).

8 Hopfgartner G, Varesio E, Tschappat V, Grivet C, Bourgogne E, Leuthold LA. Triple quadrupole linear ion trap mass spectrometer for the analysis of small molecules and macromolecules. J. Mass Spectrom. 39, 845-855 (2004).
9 Kanu AB, Dwivedi P, Tam M, Matz L, Hill HH. Ion mobility-mass spectrometry. J. Mass Spectrom. 43, 1-22 (2008).

10 Schneider BB, Covey TR, Coy SL, Krylov EV, Nazarov EG. Chemical effects in the separation process of a differential mobility/mass spectrometer system. Anal. Chem. 82, 1867-1880 (2010).

11 LeBlanc YJC, Varesio E, Hopfgartner G. Real time 2D separation; increasing the LC peak capacity using gas phase separation prior to MS analysis. Presented at: The 58th ASMS Conference on Mass Spectrometry and Allied Topics, Salt Lake City, UT, USA, 23-27 May 2010. 